\title{
Use of membrane separation device for extraction of intracellular protein and DNA
}

\author{
Yu Shi ${ }^{a}$, Xiujie Liu ${ }^{b}$, Rongji Dai ${ }^{c^{*}}$, and Yulin Deng ${ }^{d}$ \\ School of Life Science, Beijing Institute of Technology, Beijing 100081, China \\ a email: sybit2011@163.com, ${ }^{\mathrm{b}}$ email: liuxiujie0210@163.com, ${ }^{\mathrm{c}}$ email: dairongji@bit.edu.cn, ${ }^{\mathrm{d}}$ email: \\ deng@bit.edu.cn
}

\begin{abstract}
Keywords: Ultrafitration; Silica membrane; Cell; Lysis; Protein; DNA; Device
\end{abstract}
Abstract. In this paper, a membrane separation device is proposed and demonstrated to be able to lyse cells and extract intracellular protein and DNA. The device consists of cell pretreatment chamber, microfiltration unit, membrane separation unit, fluid reservoir unit and control units. The pretreatment method incorporates chemical lysis reagents and mechanical vibration induced by a micro-pump to perform cell lysis. The membrane separation module is equipped with a replaceable membrane. The ultrafiltration membrane and silica membrane are used to extract intracellular protein and DNA, respectively. The results show that the cell lysis can be achieve effectively by the pretreatment unit at the help of vibration on the membrane. The target protein was obtained using UF membrane with different molecular weight cut-off, and the silica membrane in the device had an excellent ability of extraction of intracellular DNA. The working device suggests an inexpensive, easy to be fabricated and effective method for cell lysis and extraction of intracellular protein and DNA. The resulting extraction is suitable for downstream protein and nucleic acid analysis and detection without requiring further preparation. This device can be easily integrated with other downstream components to develop a customized biological samples analytic system.

\section{Introduction}

The extraction of protein and DNA from cells is an important process in biomedicine research. In order to obtain intracellular substances such as proteins and DNA for downstream analysis, we need to first lyse cells. Cell lysis is a basic and key technique for the extraction of intracellular proteins and nucleic acids and many more for further analysis. It is widely applied in molecular biology and clinical laboratories.

Various approaches have been performed to lyse cell such as chemical reagents or enzymes to degrade cell membranes [1,2], mechanical forces to puncture the cell membranes [3], low-strength electric fields to penetrate cells [4,5], and high temperatures to disrupt cell membranes [6]. Overall, the lysis method mainly depends on cell type, samples characteristics and operation process [7]. Commonly, the efficiency of cell lysis step determined the concentration and integrity of extracted proteins and nucleic acids [8]. In most cases, the intracellular materials are very limited and difficult to be preserved for a long term. Therefore, we required to some special designs to lyse cells rapidly and productively for a high concentration, the minimum loss of extracted intracellular materials.

Currently, the methods of protein or DNA extraction mainly include phase separation and precipitation using guanidinium salts with organic solvents [9,10] and using extraction kits [11]. Some lysis devices and approaches at the microchip level often consume much time on the design and fabrication, and lack of the process of cell washing. Moreover, the cell sample quantity in the processing limits the applications in many situation which anticipates to obtain large amount samples. However, these methods often require long processing time, great labor intensity, and some hazardous chemicals. Obviously, further improvement on handling time, sample recovery, and automation is desired for extraction methods.

Here, we present work toward a novel device to continuously lyse cells and extract proteins or nucleic acids using ultrafiltration membrane or silica membrane. Membrane separation process is an important technology in separation science [12]. Particularly, ultrafiltration (UF) driven by pressure, 
is widely used in the extraction of protein from different biological samples [13]. UF membranes, with different molecular weight cut-off (MWCO), are an excellent alternative to isolate protein [14]. However, the membrane fouling is an unavoidable problem during the UF processes. In this study, we proposed a method to settle the blocked membrane using mechanical vibration exerted on the surface of UF membrane. On the other hand, silica membranes has been used to adsorb and bind DNA in the condition of chaotropic salts (guanidine salts) at lower PH (5.0-6.5) [15]. This improved method is now commercially available for the purification of DNA in biological samples. Our device was equipped with a changeable membrane material, which can be replaced according to the different extractions.

The proposed method for cell lysis and extraction of protein or DNA is easy to be implemented and integrated with other functional components to form a systematic equipment in application of molecular biology and clinical laboratories.

\section{Experimental Section}

Reagents and materials. RIPA lysis buffer, PMSF, proteinase K, RNase A, 0.25\% trypsin-EDTA, DNA extraction buffers and BCA reagent kit were purchased from Solarbio Co., Ltd., China. PBS buffer (PH 7.4) were obtained from our laboratory. Sodium hydroxide is purchased from Beijing chemical reagent company. 3, 5-dinitrosalicylic acid, potassium sodium tartrate and crystalline phenol were provided by J\&K Co., Ltd., China. Deionized water is obtained from Mill-Q water equipment in our lab. Microfiltration membrane and ultrafiltration membrane were purchased from Merck Millipore, Ltd., USA. Silica membrane was obtained from Lifefeng Co., Ltd., Hangzhou, China. Jurkat cells and SH-SY5Y cells are used in the experiments.

Design of the device. The cell washing, lysis and intracellular protein or DNA separation approaches were successfully integrated in the presented device. The device consists of cell pretreatment chamber, microfiltration unit, membrane separation unit, fluid reservoir unit and control units. The device design is shown schematically in Figure 1.

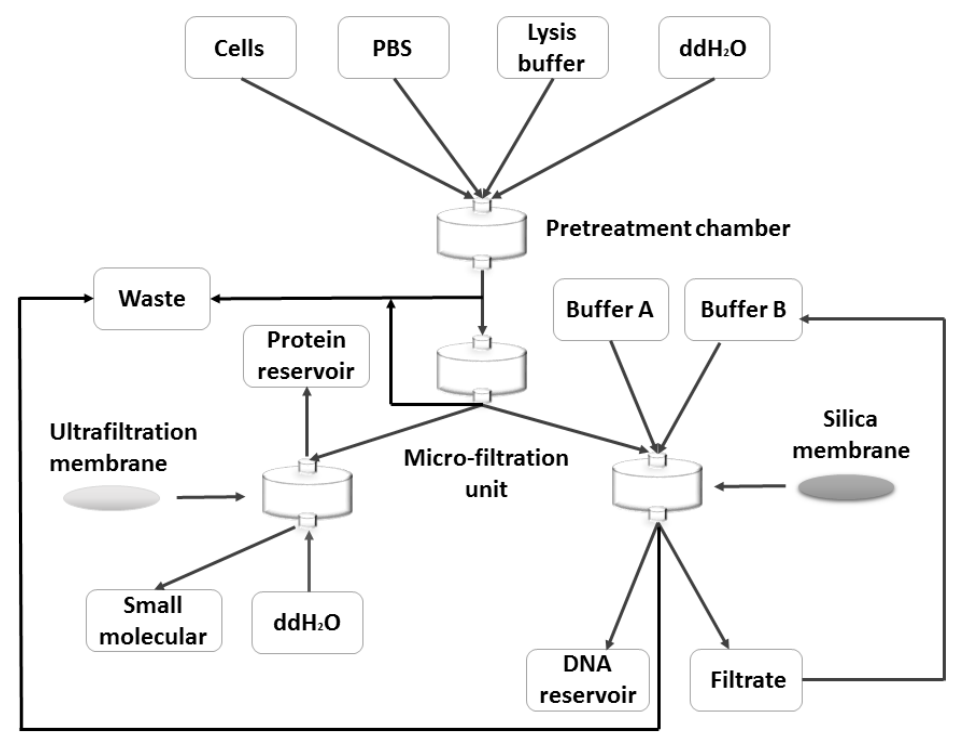

Fig. 1. Block diagram of the device system.

The functional modules comprise cell pretreatment chamber, filtration chamber and ultrafiltration unit. The device inlets were the reservoirs of cell samples, different buffers, cell washing buffer, deionized water, respectively. The outlets included waste liquid bag and the reservoirs of small molecules, protein and DNA. The temperature zone $\left(4^{\circ} \mathrm{C}\right)$ was provided for the cell pretreatment. Cells can be harvested from an upstream cell culture module at any desired state in the cultivation cycle. The cell pretreatment chamber was designed with a micro-filtration membrane (pore size, $5 \mu \mathrm{m}$; CA) which can intercept cell samples and wash cells. In this unit, the driving force and controlling of 
cell washing and lysis were driven by the micro-pumps and switch valves. In order to enhance the lysis efficiency and avoid membrane fouling, a mechanical vibration induced by pumps was introduced on the surface of membranes in cell pretreatment chamber and ultrafiltration chamber. The micro-filtration unit (pore size, $0.45 \mu \mathrm{m}$; PES) was to remove cell fragments and particles after cell lysis. Finally, all the intracellular substances (proteins or DNA) will be pumped to the ultrafiltration unit or silica membrane (pore size, $1 \mu \mathrm{m}$ ) unit for extraction of protein or DNA. The whole device is controlled by designed circuit board, which is powered by $28 \mathrm{~V} \mathrm{DC}$ and the output voltage is 12,5 and 3.3V DC.

Cell culture. Jurkat cells were cultured in RPMI 1640 medium, and SH-SY5Ycells were grown in Dulbecco's Modified Eagle Medium (DMEM). All media were supplemented with 10\% fecal calf serum (FCS) and $1 \%$ penicillin streptomycin. The cells were maintained in a humid incubator at $37^{\circ} \mathrm{C}$ with $5 \% \mathrm{CO}_{2}$.

Cell pretreatment. Jurkat cells can be injected directly through the sample reservoir. SH-SY5Y cells should be digested by trypsin before injection. The cell suspension were first pumped into the pretreatment chamber and trapped on the filter membrane $(5 \mu \mathrm{m})$. The culture medium was filtered into waste liquid bag. Then, cells were washed with PBS ( $\mathrm{pH} 7.4)$ driven by a pump for 3 times to fully remove the redundant culture medium. Subsequently, protein extraction lysis buffer $(50 \mathrm{mM}$ Tris (pH 7.4), $150 \mathrm{mM} \mathrm{NaCl}, 1 \%$ TritonX-100, $1 \%$ sodium deoxycholate, $0.1 \%$ SDS) or DNA extraction lysis buffer (100mmol Tris- $\mathrm{HCl}$ (pH8.0), 25mmol EDTA (pH 8.0), 500mmol NaCl, 1\% SDS, RNase A, proteinase K) was pumped into the pretreatment chamber. The cells were cracked with the lysis buffer. Simultaneously, the vibration was imposed on the membrane induced by switching the rotational direction of pump. The cells were fully contacted with the lysis buffer through the vibration. Finally, cell lysates were dissolved in double distilled water and the cell debris and sediments were filtered by the micro-filtration membrane $(0.45 \mu \mathrm{m})$.

Extraction of intracellular protein using ultrafiltration unit. The ultrafiltration unit was equipped with a replaceable ultrafiltration membrane (Polyethersulfone, PES). The ultrafiltration process was driven by a plunger pump. The ultrafiltration membrane (UF) can be changed for different target protein with different molecular weight cut-off (MWCO). The small molecules went through the UF and harvested in a reservoir, and proteins were collected by rinsing the UF membrane.

Extraction of intracellular DNA using silica membrane. The silica membrane had an excellent adsorbent material for extraction of DNA. When the target extraction is DNA, the silica membrane separation unit can be used to extract the intracellular DNA. The lysates were pumped to the unit equipped with a silica membrane. Silica membrane in buffer A (a high salt concentration (4M guanidine hydrochloride) at $\mathrm{pH}$ 6.0) will adsorb DNA selectively, and it will release DNA in buffer B (a low salt concentration and at $\mathrm{pH}$ 8.0). The released intracellular materials were washed with buffer A for 3 times to adsorb and accumulate DNA. The filtrate was transferred into the waste bag. Then, the buffer B was used to elute DNA from the membrane surface. The elution was added to the silica membrane chamber to elute for 3 times. Finally, the intracellular DNA was harvested in the DNA reservoir.

Determination of protein and DNA concentration. The intracellular protein was determined by BCA protein assay kit. The BCA reagent and $\mathrm{Cu}$ reagent were mixed at 50:1 (v/v) as the working liquid. BSA standard substance was diluted to a concentration of $0.5 \mathrm{mg} / \mathrm{ml}$. Then, protein samples were collected from the protein reservoir and diluted ten times. The different concentration of BSA and the protein samples were transferred into a $5 \mathrm{ml}$ centrifuge tube with $2 \mathrm{ml}$ BCA working liquid. Finally, the centrifuge tubes were dipped in a water bath at $37^{\circ} \mathrm{C}$ for $20 \mathrm{~min}$. The absorbance of the reaction liquids were measured at $562 \mathrm{~nm}$, and calculate the protein concentration according to the protein standard curve. The DNA extract $(2 \mu \mathrm{m})$ was diluted with Milli-Q water to a volume of 100 $\mu \mathrm{m}$. Then, the intracellular DNA concentration was measured at $260 \mathrm{~nm}$ by BioPhotometer plus. 


\section{Results and discussion}

Cell retention effect. The cell pretreatment chamber provided a $5 \mu \mathrm{m}$ membrane to intercept and lyse cells. Jurkat cells and SH-SY5Y cells were injected into the pretreatment chamber by peristaltic pump. The electron micrographs of jurkat cells and SH-SY5Y cells before and after filtration by a $5 \mu \mathrm{m}$ microfiltration membrane are shown in Figure 2. The results show that the membrane can effectively retain cells on the membrane surface. None cells went through the membrane into the filtrate. The cellulose acetate (CA) membrane is an excellent membrane with low adsorption, high flow rate and thermal stability. Therefore, the membrane in cell pretreatment chamber had an excellent property of cell retention.

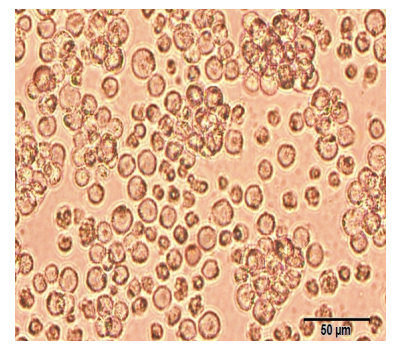

(A-a)

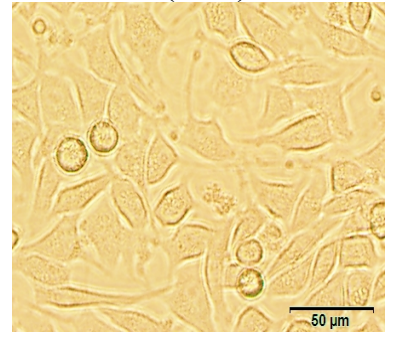

(B-a)

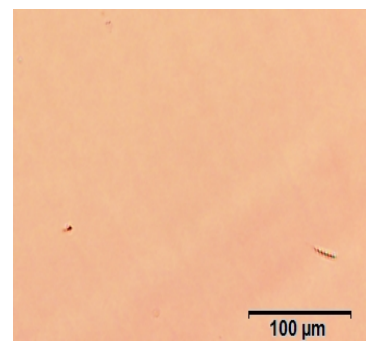

(A-b)

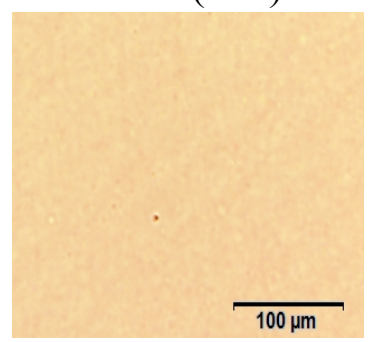

(B-b)

Fig. 2. Electron micrographs of jurkat cells (A) and SH-SY5Y cells (B) before filtration (a) and after filtration (b) by $5 \mu \mathrm{m}$ microfiltration membrane in the pretreatment chamber.

\section{Quantitative determination of intracellular proteins and DNA}
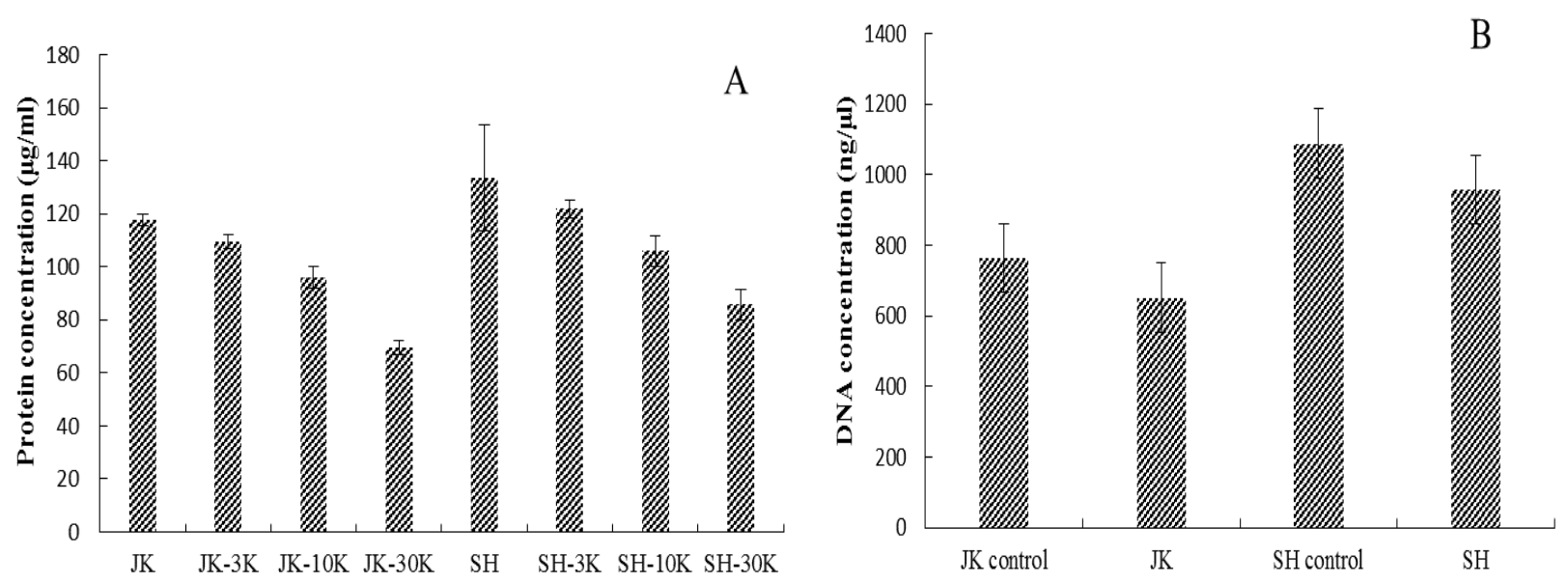

Fig. 3 The concentration of intracellular protein (A) and DNA (B) in jurkat cells and SH-SY5Y cells. $\mathrm{JK}$ and $\mathrm{SH}(\mathrm{A})$ indicate the quantitative determination of proteins by $\mathrm{BCA}$ kit. $3 \mathrm{~K}, 10 \mathrm{~K}, 30 \mathrm{~K}$ represent different molecular weight cut-off. JK and SH (B) indicate the DNA concentration harvested by the silica membrane. Controls represent the determination of DNA by DNA extraction kit.

In order to assess the properties of the membrane separation device, the intracellular protein and DNA were collected and determined. Figure 3 shows the concentration of intracellular protein and DNA in jurkat cells and SH-SY5Y cells by different methods. The protein concentrations in Figure $3 \mathrm{~A}$ show the correlation between total protein and the protein harvested by different molecular weight 
cut-off. The results indicated that the protein concentration reduced with the increasing of the membrane molecular weight cut-off. Therefore, we can obtain the target protein using UF membrane with different molecular weight cut-off. The intracellular DNA concentration using silica membrane is shown in Figure 3B. The intracellular DNA extracted by kit were 765.27 and $1088.48 \mathrm{ng} / \mu 1$, respectively. The DNA concentration extracted by silica membrane in the device were 651.7 and $958.52 \mathrm{ng} / \mu \mathrm{l}$, respectively. The DNA recovery was more than $85.16 \%$ using the device. The resulting extraction is suitable for downstream protein and nucleic acid analysis and detection without requiring further preparation.

\section{Summary}

A novel cell lysis method was proposed for automated membrane separation devices using several functional units. We have successfully demonstrated the feasibility of the device. It is experimentally determined that the protein and DNA can be obtained at a high recovery using the methods of ultrafiltration and silica membrane adsorption. Above all, this device can be easily integrated with other downstream components to develop a customized biological samples analytic system.

\section{Acknowledgements}

This research was supported by the national major scientific instruments development projects (No. 2012YQ040140).

\section{References}

[1] D. Irimia, R.G. Tompkins, M. Toner, Anal. Chem. 76 (2004) 6137-6143.

[2] R.N. Zare, S. Kim, Annu. Rev. Biomed. Eng. 12 (2010) 187-201.

[3] J. Kim, M. Johnson, P. Hill, B.K. Gale, Integr. Biol. 1 (2009) 574-586.

[4] H.Y. Wang, A.K. Bhunia, C.A. Lu, Biosens. Bioelectron. 22 (2006) 582-588.

[5] G. Mernier, N. Piacentini, T. Braschler, N. Demierre, P. Renaud, Lab Chip 10 (2010) 2077-2082.

[6] N. Privorotskaya, Y.S. Liu, J. Lee, H. Zeng, J. A. Carlisle, A. Radadia, L. Millet, R. Bashir, W.P. King, Lab Chip 10 (2010) 1135-1141.

[7] M.M. Packard, E.K. Wheeler, E.C. Alocilja, M. Shusteff, Diagnostics 3 (2013) 105-116.

[8] H. So, K. Lee, Y.H. Seo, N. Murthy, A.P. Pisano, ACS Appl. Mater. Inter. 6 (2014) 6993-6997.

[9] D. Akopian, J.D. Medh, Biotechniques 41 (2006) 426-430.

[10] R.H. Butt, T.A. Pfeifer, A. Delaney, T.A. Grigliatti, W.G. Tetzlaff,; J.R. Coorssen, Mol. Cell. Proteomics 6 (2007) 1574-1588.

[11] S.M.J. Morse, G. Shaw, S.F. Larner, Biotechniques 40 (2006) 54-58.

[12] D. Delaunay, M. Rabiller-Baudry, J.M. Gozálvez-Zafrilla, B. Balannec, M. Frappartc, L. Paugam, Chem. Eng. Process. 47 (2008) 1106-1117.

[13] N. Diagne, M. Rabiller-Baudry, L. Paugam, J. Membrane Sci. 425-426 (2013) 40-47.

[14] I. Levitsky, A. Duek, R. Naim, E. Arkhangelsky, V. Gitis, Chem. Eng. Sci. 69 (2012) 679-683.

[15] R. Boom, C.J. Sol, M.M. Salimans, C.L. Jansen, P.M. Wertheim-van Dillen, J. van der Noordaa, J. Clin. Microbiol. 28 (1990) 495-503. 